\title{
Implementación de un programa de estimulación cognitiva en personas con demencia tipo Alzheimer: un estudio piloto en chilenos de la tercera edad*
}

\author{
Implementation of a Cognitive Stimulation Program for People with \\ Alzheimer Disease: A Pilot Study in a Chilean Elderly Sample
}

Recibido: agosto 28 de 2011 | Revisado: febrero 19 de 2011 | Aceptado: julio 18 de 2012

\author{
Claudia Miranda-CASTILlO ** \\ FRANCO MASCAYANO TAPIA *** \\ ANDREA ROA HERRERA **** \\ FranCO MARAY GHIGLIOTTO ****** \\ LUCIANA SERRAINO GUERRA ******* \\ Universidad de Valparaíso, Chile
}

doi:10.11144/Javeriana.UPSY12-2.ipec

Para citar este artículo: Miranda-Castillo, C., Mascayano, F., Roa, A., Maray, F. \& Serraino, L. (2013). Implementación de un programa de estimulación cognitiva en personas con demencia tipo Alzheimer: un estudio piloto en chilenos de la tercera edad. Universitas Psychologica, 12(2), 445-455.

* Agradecemos a los adultos mayores y al personal del Hogar San Alberto Hurtado que gentilmente accedieron a participar en esta investigación y al Proyecto Fondecyt \# 11100457.

** Profesor Adjunto. Escuela de Psicología, Facultad de Medicina, Universidad de Valparaíso. E-mail: clmirandac@gmail.com

**** Investigador de la Escuela de Salud Pública, Facultad de Medicina, Universidad de Chile. E-mail: franco.mascayano@gmail.com

***** Universidad de Valparaíso. E-mail: roa.andrea@ gmail.com

${ }^{* * * * * *}$ Universidad de Valparaíso. E-mail: franco.maray@gmail.com

******** Universidad de Valparaíso. E-mail: luciana.serraino@gmail.com
RESUMEN

La Terapia de Estimulación Cognitiva (TEC) es una intervención psicosocial para personas con demencia que ha obtenido buenos resultados en población anglosajona. El objetivo de este estudio fue evaluar la eficacia de la TEC en una muestra de personas chilenas. Participaron 22 pacientes con diagnóstico de Enfermedad de Alzheimer (EA) en fase leve a moderada, pertenecientes a un centro asistencial de la ciudad de Quillota, Chile. Para determinar la eficacia del programa, se definió un diseño experimental mixto $2 \times 2$ de medidas repetidas, asignándose aleatoriamente la muestra a dos grupos: intervención (GI) y control (GC). Se establecieron tres dimensiones para evaluar: cognición, calidad de vida y capacidad funcional de la persona con demencia. Posterior a la intervención, el GI presentó una mejoría significativa en sus indicadores de deterioro cognitivo y calidad de vida; por el contrario, el GC no evidenció cambios positivos en ninguna dimensión, empeorando el declive cognitivo. Ninguno de los grupos experimentó cambios significativos en la capacidad funcional. Si bien en la comparación intergrupo no existieron diferencias significativas en ninguna variable, el GI presentó una mejoría sustancial de la función cognitiva, en comparación con el aumento en la progresión del deterioro cognitivo en el GC. Se recomienda que futuras investigaciones contrasten dichos resultados utilizando una muestra más grande de personas con EA.

Palabras clave autores

Demencia, enfermedad de Alzheimer, estimulación cognitiva, Latinoamérica.

Palabras clave descriptores

Anciano, estudio piloto, Chile.

\section{A B S T R A C T}

Cognitive Stimulation Therapy (CST) is a psychosocial intervention for people with dementia that has been successful in Anglo-Saxon population. The aim of this study was to assess the effectiveness of CST in a sample of Chilean elders. The participants were 22 patients diagnosed with Alzheimer's disease (AD) in mild to moderate stage, who were living in a residential care home in the town of Quillota, Chile. In order to determine the effectiveness of the program, an experimental mixed design $2 \times 2$ (repeated measures) was employed. The sample was randomly assigned into two 
groups: intervention group (IG) and control group (CG). Three dimensions were assessed: cognition, quality of life and functional ability. After the intervention, the IG presented a significant improvement in their cognitive function and quality life. In contrast, the CG did not show positive changes in any dimension. In fact, CG showed a worsening in its cognitive deterioration. Both groups did not showed significant changes in functional ability. Even though intergroup comparisons did not yield significant differences, the IG showed an important improvement in cognitive function, compared with an increase in the progression of cognitive impairment in the CG. It is recommended that future research will contrast these results using a larger sample of people with AD. Key words authors

Dementia, Alzheimer's Disease, Cognitive Stimulation, Latin America.

Key words plus

Elderly, Pilot Study, Chile.

\section{Introducción}

De acuerdo con Oliver, Navarro, Meléndez, Molina y Tomás (2009), la demencia es un síndrome que se caracteriza por graves alteraciones de las capacidades funcionales, emocionales y cognoscitivas, las cuales conducen a diversos grados de discapacidad. Por ende, actualmente se conceptualiza como un problema de vital importancia en la etapa de la vejez (Gil \& Martín, 2006). Un estudio transcultural realizado por Sousa et al. (2009), plantea que, por lo menos en países con menos recursos, la demencia sería la mayor causa de discapacidad en las personas longevas. En el caso de Chile, se estima que más de 100.000 personas presentan en la actualidad una demencia, siendo el Alzheimer una de las diez primeras causas de muerte en la tercera edad (Fuentes \& Slachevsky, 2005).

Actualmente, el manejo terapéutico de la demencia se enfoca principalmente en el tratamiento farmacológico, utilizando básicamente sustancias procolinérgicas (donepezilo y la galantamina) (Fuentes, Donoso, Slachevsky \& Villalobos, 2008). Sin embargo, a pesar de los resultados favorables (Olin \& Schneider, 2002; Whitehead et al., 2004), la terapia farmacológica ha presentado ciertas limitaciones clínicas, cuestionamientos en su eficacia terapéutica a largo plazo en ámbitos cognoscitivos, emocionales y conductuales y, además, resultados de "costo-beneficio" desfavorables (Ballard, Sorensen \& Sharp, 2007; Spector, Woods \& Orrell, 2010; Sramek \& Cutler, 1999), por lo cual se considera como una terapia insuficiente, si es que no se acompaña de terapia no farmacológica (Deus, 2006).

Considerando los antecedentes señalados, y con el fin de constituir tratamientos de corte más psicosocial para complementar la terapia farmacológica, ciertos autores comenzaron a diseñar, en la segunda mitad del siglo XX, programas terapéuticos encaminados a compensar o restaurar los ámbitos afectados por el síndrome demencial (Peña-Casanova, 1999). Dichas intervenciones tienen como eje principal el proceso de "estimulación cognitiva", definida como un conjunto de tareas y actividades generadas que buscan potenciar las principales funciones cognoscitivas (Uzzell \& Gross, 1986), además de fomentar y mejorar ciertos factores psicosociales, como el "sentido de pertenencia", motivación, socialización y modularidad afectiva (Cantegreil-Kallen, De Rotrou \& Rigaud, 2009).

En la actualidad, se ha acumulado amplia evidencia con respecto a la efectividad de las intervenciones psicosociales en demencia (Olazarán et al., 2010). En sus diferentes aplicaciones, los abordajes psicosociales, y en particular la estimulación cognitiva, han exhibido resultados favorables para fortalecer y mejorar la calidad de vida, la interacción social, la comunicación y la cognición de los participantes (Moniz-Cook, Vernooij-Dassen, Woods \& Orrell, 2011; Woods, Aguirre, Spector \& Orrell, 2012), particularmente si el tratamiento se mantiene por largo plazo (Vernooij-Dassen, Vasse, Zuidema, Cohen-Mansfield \& Moyle, 2010). La efectividad de los abordajes psicosociales radica en que se entiende al paciente como un sujeto biopsico-social, de forma que la intervención que se lleva a cabo en este ámbito repercute positivamente en su entorno social, familiar, entre otros, además de tratar sus alteraciones cognitivas y comportamentales (Escarabajal, 2010).

Concordante con lo anterior, se ha constatado una creciente inclusión de las intervenciones psicosociales en las políticas públicas y los objetivos sanitarios de diversos países, materializándose en 
la incorporación de tales abordajes a las recomendaciones de guías clínicas de demencia de Europa (Vasse et al., 2012), Estados Unidos (Rabins et al., 2007) y Canadá (Hogan et al., 2008). No obstante, dicho proceso en Latinoamérica ha sido lento e improductivo a la fecha, debido principalmente a la escasez de investigaciones clínicas que validen su aplicación en dicha población.

Dentro del paradigma de los abordajes psicosociales en demencia, un grupo de autores ingleses elaboró un tipo de tratamiento llamado Terapia de Estimulación Cognitiva (TEC) (Orrell, Spector, Thorgrimsen \& Woods, 2005; Spector et al., 2003; Woods, Thorgrimsen, Spector, Royan \& Orrell, 2006). Dicha intervención, que encuentra sus antecedentes en programas previos de estimulación cognitiva (Breuil et al., 1994), en la terapia de orientación a la realidad (Zanetti et al., 1995), reminiscencia (Hala, 1975) y validación (Bleathman \& Morton, 1996), se define como un programa de apoyo y potenciación global de las personas en las etapas tempranas de la demencia que abarcaría dimensiones como la cognición, afectividad, relaciones interpersonales, comunicación y lenguaje (Spector, Thorgrimsen, Woods $\&$ Orrell, 2006).

En los primeros ensayos clínicos donde se aplicó la TEC (Spector, Orrell, Davies \& Woods, 2001; Spector et al., 2003), se reportaron mejorías significativas en las medidas de la función cognitiva global. También existieron diferencias importantes entre los grupos de control y de intervención, referentes a los índices de calidad de vida. Por su parte, un análisis de costo-beneficio (Knapp et al., 2006), reveló que la TEC es relativamente barata y más rentable que el tratamiento farmacológico habitual, lo cual estaría ligado a la disminución del deterioro cognitivo.

Considerando los antecedentes señalados, se realizó en un grupo de ancianos chilenos una aplicación piloto de la TEC (Spector et al., 2006), con el objetivo de, por una parte, adaptar el programa terapéutico a la realidad chilena y, por otra, evaluar de manera rigurosa su efectividad en ancianos chilenos con enfermedad de Alzheimer. Se hipotetizó que las personas que participaran en el grupo de tratamiento exhibirían mejorías significativas en sus índices de deterioro cognitivo y percepción de calidad de vida, en comparación con los individuos que no recibieran tratamiento alguno.

\section{Método}

\section{Participantes}

Se contactó más de 20 centros geriátricos, casas de reposo y residenciales para ancianos, buscando una población estable dentro de la cual pudiesen constatarse cuadros con diagnóstico de demencia tipo Alzheimer, lográndose un grupo inicial de 28 ancianos todos residentes en el Hogar para el Anciano "Padre Alberto Hurtado" de Fundación las Rosas (ubicado en la región de Valparaíso, Chile).

Para ser incluidos en la investigación, los participantes, personas de ambos sexos y mayores de 65 años, debieron cumplir con los siguientes criterios:

a. Cumplir con los criterios diagnósticos del DSM-IV-TR (APA, 2000) para enfermedad de Alzheimer.

b. Puntuar entre 10-24 puntos en el Minimental State Examination (MMSE) (adaptación de Quiroga, Albala \& Klaasen, 2004).

c. Contar con capacidad visual y auditiva preservada, para de ese modo participar óptimamente en las actividades programadas.

d. Carecer de impedimentos físicos que dificultasen el trabajo adecuado en las actividades planificadas.

e. No presentar diagnóstico asociado de dificultades de aprendizaje.

Tomando en cuenta estos criterios, del total de 28 personas consideradas en instancia preliminar, tres fueron eliminadas por no cumplir con el criterio "c", dos por reticencia a participar en el programa de actividades y uno debido a dificultades asociadas a enfermedad médica, logrando así una muestra final de 22 personas, conformada por 14 mujeres y 8 hombres $(M=83.6 ; D E=9.76)$. 


\section{Procedimiento}

El diseño utilizado, caracterizado como diseño cuasiexperimental mixto $2 \times 2$ (de medidas repetidas), se desarrolló en dos instancias. En primer lugar, tras la obtención de consentimiento informado, se llevó a cabo la división de la muestra en dos grupos: 11 personas fueron asignadas aleatoriamente al grupo de intervención (GI) que recibió la TEC, por un tercero no involucrado con posterioridad al estudio, y 11 al grupo control (GC). Ambos grupos fueron sometidos luego a evaluaciones con el fin de determinar sus niveles basales en los ámbitos cognitivo, de calidad de vida y funcionalidad global.

Posteriormente, tras siete semanas en las cuales se llevaron a cabo las 14 sesiones de terapia de estimulación cognitiva (TEC) en el GI (con un plan de trabajo de 2 sesiones semanales de duración aproximada de 45 minutos cada una), se condujo una segunda evaluación al total de la muestra. Dicha medición final fue realizada por evaluadores ciegos con desconocimiento del grupo al cual fue asignado cada participante del estudio.

\section{Terapia de Estimulación Cognitiva (TEC)}

La TEC es un tratamiento psicosocial breve, sencillo y didáctico para personas con demencia de leve a moderada. Aborda una serie de temáticas generales como a) actividad física, b) identificación de sonidos, c) exploración de infancia, d) denominación de alimentos, e) discusión sobre actualidad, f) identificación de caras/escenas, g) asociación de palabras, h) potenciación de la creatividad, i) categorización de objetos, j) desarrollo del sentido de orientación, k) utilización de dinero, l) juegos con números, $\mathrm{m}$ ) juegos con palabras y n) pruebas grupales. Su desarrollo se realiza en 14 sesiones, dos por cada semana. Cada sesión tiene una duración de aproximadamente 45 minutos, permitiendo algunos minutos finales para la reflexión personal de sus participantes. Todas las sesiones cuentan con una actividad inicial "de calentamiento" y otra de cierre, además de dos actividades principales según la temática que se desarrolle (Spector et al., 2010). Las sesiones se caracterizan por una estimulación multisensorial, en donde los participantes realizan tareas como hacer coincidir sonidos comunes e imágenes. Por otro lado, también se integran aspectos de reminiscencia y orientación, por ejemplo, a través de la comparación de monedas antiguas y nuevas con sus respectivos valores.

\section{Instrumentos}

\section{Cognición}

Con el fin de evaluar y obtener un diagnóstico grueso del deterioro asociado a las funciones cognitivas básicas, se realizó una primera medición haciendo uso del Mini Mental State Examination (MMSE) (Quiroga et al., 2004). Su utilización se sustentó en su simplicidad y brevedad de aplicación, además de su reconocida validez y confiabilidad en la cuantificación de funciones cognitivas (Cockrell \& Folstein, 2002; Zamarrón, Tárraga \& Fernández, 2008), enmarcándolo como el instrumento más utilizado para evaluar los efectos de intervenciones en pacientes con demencia así como para valorar la progresión del deterioro cognitivo (Lezak, 2004).

La segunda medición de funciones cognitivas se llevó a cabo mediante el Alzheimer's Disease Assesment Scale-Cognitive ([ADAS-Cog]; Rosen, Mohs \& Davis, 1984), que ha comprobado una alta consistencia evaluativa, además de una alta correlación asociada a mediciones realizadas por medio del MMSE (diferenciándose de este en el carácter creciente de las puntuaciones que se obtienen al presentarse aumento del deterioro cognitivo). También, debido a su utilización en diversos estudios farmacológicos (Knapp et al., 1994), representa uno de los instrumentos más sensibles de medición geriátrica y de comparación con tratamientos combinados de medicamentos y programas psicosociales. En el caso de este instrumento, puntajes más altos indican mayor deterioro cognitivo.

\section{Calidad de vida}

Para evaluar esta variable se utilizó la Quality of Life-Alzheimer Disease Scale ([QoL-AD]; Logsdon, Gibbons, McCurry \& Teri, 1999). Este instrumento 
cuenta con 13 ítems que abarcan la percepción de aspectos tales como salud física, nivel de energía, estado de ánimo, memoria, situación familiar, amistades, diversión, situación económica, etc. Cuenta con las ventajas de un lenguaje simple y una aplicación rápida, además de un sistema de evaluación adicional por parte del cuidador. Para efectos de esta investigación, se tradujo y adaptó del inglés al español para su posterior aplicación.

\section{Nivel de funcionalidad}

El instrumento implementado para esta variable fue el Índice de Barthel [IB] (Mahoney \& Barthel, 1965). Consta de 10 ítems, que miden la dependencia de ayuda en aspectos de movilidad y de cuidado personal tales como alimentación, desplazamiento y otros, en un rango global que puede variar entre 0 puntos (completamente dependiente) y 100 puntos (completamente independiente) (Cid-Ruzafa \& Damián-Moreno, 1997). Se utilizó debido a su simplicidad y rapidez de aplicación, así como por los beneficios asociados a una alta confiabilidad constatada tanto en evaluaciones inter como intraobservador (Loewen \& Anderson, 1988).

\section{Análisis de datos}

Los datos fueron analizados utilizando el Statistical Package for Social Sciences (SPSS, 2008), versión 17. En base al análisis de normalidad de la muestra (prueba Kolmorogov-Smirnov y análisis de homogeneidad de varianzas de Levene) y su correspondiente distribución normal se sometieron los datos a las pruebas $t$ de Student de comparación de grupos, chi cuadrado y análisis de varianza (ANOVA) de Modelo Mixto para Medidas Repetidas, estableciéndose un nivel de significancia de $p<0.05$.

En detalle se llevaron a cabo los siguientes análisis:

1. Comparación intergrupo de variables sociodemográficas y clínicas preintervención.

2. Evaluación del efecto de la intervención en los GC y GI.

TABLA 1

Resumen de variables sociodemográficas y clínicas de la muestra

\begin{tabular}{|c|c|c|c|c|c|c|c|}
\hline & & \multicolumn{2}{|c|}{ GC } & \multicolumn{2}{|c|}{ GI } & \multirow{2}{*}{ Est. } & \multirow{2}{*}{$p$} \\
\hline & & $N$ & $\% / M(D E)$ & $N$ & $\% / M(D E)$ & & \\
\hline Género & $\begin{array}{l}\text { Hombre } \\
\text { Mujer }\end{array}$ & $\begin{array}{l}4 \\
7\end{array}$ & $\begin{array}{l}36.4 \\
63.6\end{array}$ & $\begin{array}{l}4 \\
7\end{array}$ & $\begin{array}{l}36.4 \\
63.6\end{array}$ & $\chi^{2}=0.2$ & 0.665 \\
\hline Edad & & 11 & $83.3(10.3)$ & 11 & $84(9.6)$ & $t=0.149$ & 0.883 \\
\hline Años de Institución & & 11 & $1.7(1.1)$ & 11 & $2(1.1)$ & $t=0.582$ & 0.567 \\
\hline Estado Civil & $\begin{array}{c}\text { Soltero } \\
\text { Separado } \\
\text { Viudo } \\
\end{array}$ & $\begin{array}{l}7 \\
0 \\
4 \\
\end{array}$ & $\begin{array}{c}63.6 \\
0 \\
36.4 \\
\end{array}$ & $\begin{array}{l}3 \\
1 \\
7 \\
\end{array}$ & $\begin{array}{c}27.3 \\
9.1 \\
63.6 \\
\end{array}$ & $\chi^{2}=8.3$ & $0.016^{*}$ \\
\hline Escolaridad & $\begin{array}{c}\text { Univ. } \\
\text { Técnico } \\
\text { Ed. Media } \\
\text { Ed. Básica }\end{array}$ & $\begin{array}{c}0 \\
1 \\
0 \\
10\end{array}$ & $\begin{array}{c}0 \\
9.1 \\
0 \\
91.9 \\
\end{array}$ & $\begin{array}{c}1 \\
0 \\
0 \\
10\end{array}$ & $\begin{array}{c}9.1 \\
0 \\
0 \\
91.9 \\
\end{array}$ & $x^{2}=2$ & 0.368 \\
\hline Rendimiento Cognitivo & $\begin{array}{c}\text { MMSE } \\
\text { ADAS-Cog }\end{array}$ & $\begin{array}{l}11 \\
11 \\
\end{array}$ & $\begin{array}{l}18.9(4.1) \\
23.2(7.9)\end{array}$ & $\begin{array}{l}11 \\
11 \\
\end{array}$ & $\begin{array}{l}19.1(3.8) \\
25.4(8.9)\end{array}$ & $\begin{array}{l}t=0.1 \\
t=0.6\end{array}$ & $\begin{array}{l}0.916 \\
0.543\end{array}$ \\
\hline Calidad de Vida & QoL-AD & 11 & $30.6(4.3)$ & 11 & $28.2(5.9)$ & $t=-1.1$ & 0.276 \\
\hline Nivel Funcional & IB & 11 & $60.5(33.4)$ & 11 & $63.6(29.2)$ & $t=-1.1$ & 0.267 \\
\hline
\end{tabular}

$* p<0.05 ; \mathrm{GC}=$ grupo control; GI = grupo de intervención; Est. = análisis estadístico utilizado; $N=$ número de integrantes; $M$ = media; $D E=$ desviación estándar; MMSE = Mini Mental State Examination; ADAS-Cog = Alzheimer's Disease Assessment Scale - Cognition; QoL-AD = Quality of Life - Alzheimer's Disease; IB = Índice de Barthel.

Fuente: elaboración propia. 


\section{Resultados}

\section{Características basales de los participantes}

La Tabla 1 muestra las características sociodemográficas y clínicas de la muestra previa realización de la TEC. Los participantes se encontraban en un rango etario de 65 a 95 años $(M=83.68$ años; $D E=9.76)$, habiendo 14 mujeres $(M=87.38$ años; $D E=8.08)$ y 8 hombres $(M=78.33$ años; $D E=9.87)$, tanto solteros $(45.5 \%)$ como casados $(4.5 \%)$ y viudos $(50 \%)$, todos institucionalizados por un período entre 1 y 3 años $(M=1.86$ años; $D E=1.08)$ y con un nivel medio de escolaridad correspondiente a preparatoria o nivel básico para más del $90 \%$ del grupo, presentándose solo dos participantes con estudios de nivel superior.

De todas estas variables, solo se constató una diferencia significativa en la correspondiente a estado civil, habiendo más solteros en el GC y más viudos en el GI $\left(\chi^{2}=8.3 ; p=0.016\right)$.

En cuanto al análisis de las variables clínicas - cognición, calidad de vida y nivel de funcionalidad global- aun presentándose variaciones de puntuación entre ambos grupos, estas no alcanzaron márgenes significativos en ninguna de las mediciones efectuadas. Particularmente, se apreció que el nivel de deterioro cognitivo basal para ambos grupos se caracterizó entre los niveles de leve a moderado, en porcentajes del $63.6 \%$ y $36.3 \%$, respectivamente para GC, y del $45.5 \%$ y $54.5 \%$ para GI.

\section{Efectos de la Terapia de Estimulación Cognitiva}

Como puede apreciarse en la Tabla 2 y en la Figura 1, tras el período de 14 sesiones de estimulación cognitiva, se encontraron diversas variaciones en todas las variables tanto para los participantes de GC como para los de GI.

\section{Rendimiento cognitivo}

En relación con el desempeño asociado a las funciones cognitivas tanto gruesas (medidas por el
MMSE) como específicas (medidas por el ADASCog), se observó un efecto de interacción significativo entre las mediciones pre-post y el recibir o no la intervención. De esta manera, contrariamente a lo que sucedió con el GC que mantuvo su progresión de deterioro (ver Figura 1), las personas del GI incrementaron su rendimiento cognitivo significativamente en todos los niveles evaluados $(F(1,20)=9.51, p<0.01)$ como del ADAS-Cog $(F(1,20)=4.56, p<0.05)$. Por otra parte, no se observaron efectos principales en el rendimiento cognitivo tanto a nivel intragrupal pre-post (MMSE: $F(1,20)=1.05, p>0.05$; ADAS-Cog: $F(1,20)=0.39, p>0.05)$ como a nivel intergrupal (MMSE: $F(1,20)=0.83, p>0.05$; ADAS-Cog: $F(1,20)=0.06, p>0.05)$.

\section{Calidad de vida}

Solo se observó un efecto principal significativo intragrupal que muestra que la calidad de vida aumentó significativamente en el GI $(F(1,20)=6.07$, $p<0.05)$. Además, se encontró una tendencia a un efecto de interacción entre las mediciones pre-post de calidad de vida y el recibir o no la intervención. Sin embargo, dicho efecto no alcanzó la significancia estadística $(F(1,20)=4.09, p=0.057)$.

TABLA 2

Descripción de variables clínicas al inicio y tras seguimiento para GC y GI

\begin{tabular}{llrrrl}
\hline & & \multicolumn{4}{c}{ Evaluación } \\
\cline { 3 - 6 } & & \multicolumn{2}{c}{ Inicial } & \multicolumn{2}{c}{ Final } \\
\cline { 3 - 6 } & & M & DE & M & \multicolumn{1}{c}{ DE } \\
\hline \multirow{2}{*}{ MMSE } & GC & 18.9 & 4.1 & 17.9 & 5.4 \\
& GI & 19.0 & 3.8 & 21.1 & 4.3 \\
\hline \multirow{2}{*}{ Adas-Cog } & GC & 23.1 & 7.9 & 25.3 & 11.5 \\
& GI & 25.4 & 8.9 & 21.4 & 7.7 \\
\hline \multirow{2}{*}{ QoL-AD } & GC & 30.6 & 4.2 & 31.1 & 3.5 \\
& GI & 28.1 & 5.8 & 33.7 & 5.9 \\
\hline \multirow{2}{*}{ IB } & GC & 60.4 & 33.4 & 56.8 & 36.2 \\
& GI & 63.6 & 29.2 & 61.8 & 25.7 \\
\hline
\end{tabular}

Fuente: elaboración propia. 


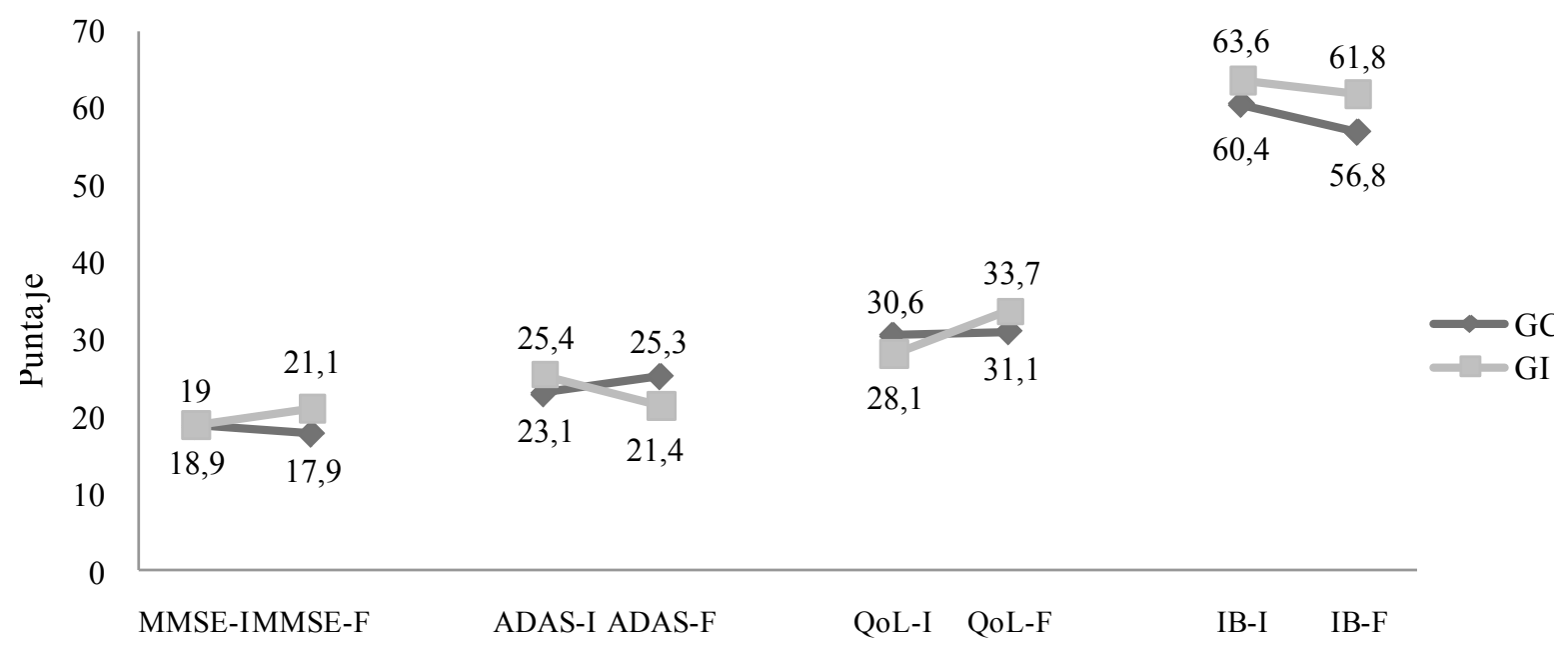

Instrumento

Figura 1. Comparación de medias de variables clínicas para GC y GI al inicio y tras seguimiento. Fuente: elaboración propia.

\section{Nivel de funcionalidad}

No se encontró ningún efecto significativo en el nivel de funcionalidad tanto a nivel intragrupal $(F(1,20)=2.08, p>0.05)$, intergrupal $(F(1,20)$ $=0.09, p>0.05)$ y de interacción $(F(1,20)=0.23$, $p>0.05)$.

$\mathrm{GC}=$ grupo control; GI = grupo de intervención; MMSE-I = Mini Mental State Examination - medición inicial; MMSE-F = Mini Mental State Examination - medición final; ADAS-I = Alzheimer's Disease Assessment Scale - Cognition medición inicial; ADAS-F $=$ Alzheimer's Disease Assessment Scale - Cognition - medición final; QoLI = Quality of Life - Alzheimer's Disease - medición inicial; QoL-F = Quality of Life - Alzheimer's Disease - medición final; IB-I = Índice de Barthel-medición inicial; IB-F = Índice de Barthel-medición final.

\section{Discusión y conclusiones}

Se encontraron resultados favorables sobre las variables de rendimiento cognitivo global y calidad de vida tras la aplicación de la TEC. Dichos resultados son coincidentes con literatura previa, tanto para anteriores aplicaciones de la TEC (Spector et al., 2001; Spector et al., 2003), como para otros programas de estimulación cognitiva de estructura semejante (Bach, Bach, Böhmer, Frühwald \& Grilc, 1995; Breuil et al., 1994; Tárraga et al., 2006; Woods et al., 2012). Estos resultados positivos pueden asociarse al conjunto de distintas técnicas utilizadas dentro de la sesiones de la TEC, sustentadas en otras terapias psicosociales, que a su vez también han presentado resultados favorables, tales como la terapia de reminiscencia, terapia de validación, terapia de orientación a la realidad, estimulación cognitiva activa, etc. (Neal \& Briggs, 2003; Olazarán et al., 2010; Woods, Spector, Jones, Orrell \& Davies, 2005). Por ejemplo, las "técnicas de orientación a la realidad", deben ser consideradas un mecanismo que propicia una mejoría en cuanto a la orientación en el tiempo y espacio de las personas con demencia. Las mejorías en la orientación se traducen en una mejor comprensión y adaptación al medio que les rodea, conduciendo potencialmente a un aumento de la sensación y percepción de control, autoeficacia y autoestima (Gagnon, 1996). Por otro lado, las técnicas de "estimulación cognitiva activa" inciden en la producción del aumento en las facultades cognitivas específicas, como la atenciónconcentración, la memoria, las funciones ejecutivas, 
el lenguaje, la orientación temporoespacial, la capacidad visoespacial, el cálculo y las praxias (PeñaCasanova, 1999). Asimismo, es posible sopesar la influencia de las técnicas de reminiscencia, basadas en la estimulación a los participantes para relatar sucesos o eventos de sus particulares pasados (Butler, 2002), y de las técnicas de validación centradas en la aceptación y validación empática tanto de los sentimientos como de la realidad personal de la experiencia del otro, sobre el funcionamiento cognitivo global, de manera más genérica y, sobre la comunicación, de manera más específica (Bleathman \& Morton, 1996).

Por otra parte, el aumento significativo en la evaluación de la calidad de vida de los participantes que recibieron la TEC podría deberse al apoyo consistente entregado por la terapia a nivel de mejoría de memoria, interacción social con pares y aumento del nivel de energía ligado al aumento de la capacidad para hacer tareas (Woods et al., 2006), todo lo cual se genera fundamentalmente a partir de la modalidad de trabajo grupal, del constante incentivo de la comunicación verbal dentro del grupo, de la búsqueda de actividades placenteras para cada sesión y, además, de ítems específicos asociados a la capacidad de resolución de problemas cotidianos.

Tal como se esperaba, el estudio mostró una ausencia de cambios significativos en la variable de funcionalidad. Tales hallazgos resultan coincidentes con lo que la literatura ha señalado, ya que resulta poco probable que las modificaciones en áreas cognitivas, fundamentalmente, puedan tener impactos significativos en las áreas de funcionamiento e independencia (Woods et al., 2012; Zanetti et al., 1997; Zanetti et al., 2001).

Actualmente, no se cuenta con datos empíricos que avalen la efectividad de los programas de estimulación cognitiva en poblaciones latinoamericanas, lo que dificulta la decisión, a nivel de políticas sanitarias, de incluir esta terapia como uno de los tratamientos básicos para reducir el deterioro cognitivo progresivo. La evidencia acerca de la eficacia que puede aportar este tipo de intervenciones, permitiría visualizar alternativas válidas para manejar el deterioro cognitivo y las demencias, disminuyendo el estigma que etiqueta a las personas con demencia como individuos que deben aguardar el fin de su ciclo vital en la más absoluta pasividad. En este sentido, resultados preliminares de un estudio realizado en Chile en ancianos con demencia (Covarrubias, Madrid, Tapia \& Vásquez, 2012) mostraron que las necesidades insatisfechas más frecuentes de dicho grupo fueron la necesidad de contar con compañía (40\%), tener más actividades en qué ocupar el tiempo $(36.6 \%)$ y recibir más ayuda para mejorar la función cognitiva (30.5\%). Es interesante que, todas estas necesidades podrían satisfacerse si la persona con demencia recibiera una intervención psicosocial y particularmente estimulación cognitiva. Por consiguiente, resulta urgente la formulación e implementación de políticas públicas en América Latina que visibilicen y aborden integralmente el síndrome demencial. Dicha estrategia debe estar dirigida a fomentar la investigación, la generación de programas nacionales y locales, y la estructuración de guías clínicas que estandaricen procesos de diagnóstico y tratamiento de las diferentes demencias. Esto último permitiría validar e integrar las intervenciones psicosociales a los servicios sanitarios del país, en el marco de programas nacionales, y no como esfuerzos aislados con baja repercusión a nivel poblacional. Es menester que, tal cual señalan la World Health Organization y Alzheimer's Disease International (2012), se conceptualice a la demencia como un problema prioritario para la salud pública, que exige el compromiso mancomunado de todos los actores de los servicios de salud, de dispositivos comunitarios y de la sociedad en general, para ofrecer respeto, apoyo y asistencia a los adultos mayores con dicho diagnóstico.

\section{Limitaciones del estudio}

Si bien los instrumentos utilizados no han sido adaptados ni validados en población chilena (salvo el Mini-Mental), fueron escogidos dada su categoría de instrumentos recomendados por el European Alzheimer's Disease Consortium (EADC), para evaluar el impacto de intervenciones psicosociales en cognición y calidad de vida en pacientes con demencia (Moniz-Cook et al., 2008; Verhey et al., 2004). Se recomienda que, con el fin de generar 
indicadores de eficacia terapéutica más adecuados, futuras investigaciones tengan como objetivo validar en población chilena y/o latinoamericana cuestionarios y escalas actualmente utilizados a nivel global en el área de las demencias.

Con respecto al análisis comparativo de las variables clínicas a nivel intergrupal, no se hallaron diferencias significativas en ninguna de las variables. Esto puede deberse fundamentalmente al tamaño muestral, el cual, al ser un estudio piloto, no contó con la potencia estadística adecuada y en consecuencia podría haber generado que no fueran detectadas diferencias significativas reales en las puntuaciones entre ambos grupos. Se recomienda que próximos ensayos clínicos incluyan muestras más representativas, para comprobar los datos aquí reportados. Los resultados y conclusiones que puedan ser extraídos de dichas investigaciones, podrían convertirse en la base de futuras implementaciones de la terapia de estimulación cognitiva.

\section{Referencias}

American Psychiatric Association. (2000). Diagnostic and statistical manual of mental disorders (4a. ed. rev.). Washington, DC: Autor.

Bach, D., Bach, M., Böhmer, F., Frühwald, T. \& Grilc, B. (1995). Reactivating occupational therapy: A method to improve cognitive performance in geriatric patients. Age and Ageing, 24(3), 222-226.

Ballard, C., Sorensen, S. \& Sharp, S. (2007). Pharmacological therapy for people with Alzheimer's disease: The balance of clinical effectiveness, ethical issues and social and healthcare costs. Journal of Alzheimer's Disease, 12(1), 53-59.

Bleathman, C. \& Morton, I. (1996). Validation therapy: A review of its contribution to dementia care. British Journal of Nursing, 5(14), 866-868.

Breuil, V., De Rotrou, J., Forette, F., Tortrat, D., Ganansia-Ganem, A., Fambourt, A., et al. (1994). Cognitive stimulation of patients with dementia: Preliminary results. International Journal of Geriatric Psychiatry, 9(3), 211-217.

Butler, R. (2002). Life review. En Encyclopedia of Aging (1a. ed.). New York: Macmillan. Recuperado el 20 de marzo de 2011, de http://www.encyclopedia. com/doc/1G2-3402200232.html

Cantegreil-Kallen, I., De Rotrou, J. \& Rigaud, A. (2009). Cognitive stimulation for people with mild cognitive impairment and early dementia. En E. MonizCook \& J. Manthorpe (Eds.), Early psychosocial interventions in dementia. Evidence-based practice (pp. 81-92). Philadelphia: Jessica Kingsley Publishers.

Cid-Ruzafa, J. \& Damián-Moreno, J. (1997). Valoración de la discapacidad física: el índice de Barthel. Revista Española de Salud Pública, 71(2), 127-137.

Cockrell, J. \& Folstein, M. (2002). Mini-Mental State Examination. En J. R. M. Copeland, M. T. AbouSaleh \& D. G. Blazer (Eds.), Principles and practice of geriatric psychiatry (2a. ed., pp. 140-141). Chichester, UK: John Wiley \& Sons Ltd.

Covarrubias, F., Madrid, M., Tapia, T. \& Vásquez, K. (2012). Necesidades de las personas con demencia y su relación con factores sociales, personales y de su cuidador informal principal. Tesis inédita de Licenciatura, Universidad de Valparaíso, Valparaíso, Chile.

Deus, J. (2006). Estimulación cognitiva en demencias: eficacia o placebo. Informaciones Psiquiátricas, 184, 119-152.

Escarabajal, M. D. (2010, febrero). Intervenciones no farmacológicas en pacientes con demencia. Documento presentado en el 11을 Congreso Virtual de Psiquiatría, Universidad de Jaén, Andalucía, España. Recuperado el 12 de Marzo del 2011, de http:// www.bibliopsiquis.com/bibliopsiquis/bitstream/1 0401/935/1/16cof645476.pdf

Fuentes, P., Donoso, A., Slachevsky, A. \& Villalobos, A. (2008). Guía clínica: trastorno cognitivo y demencia en el adulto mayor. Santiago de Chile: Ministerio de Salud.

Fuentes, P. \& Slachevsky, A. (2005). Enfermedad de Alzheimer: actualización en terapia farmacológica. Revista Médica de Chile, 133(2), 224-230.

Gagnon, D. (1996). A review of reality orientation, validation therapy, and reminiscence therapy with the Alzheimer's client. Physical $\mathbb{F}$ Occupational Therapy in Geriatrics, 14(2), 61-75.

Gil, P. \& Martín, J. (2006). Demencia. En Sociedad Española de Geriatría y Gerontología (Ed.), Tratado de geriatría para residentes (pp. 173-188). Madrid: Sociedad Española de Geriatría y Gerontología. 
Hala, M. P. (1975). Reminiscence group therapy project. Journal of Gerontological Nursing, 1(3), 34-41.

Hogan, D. B., Bailey, P., Black, S., Carswell, A., Chertkow, H., Clarke, B., et al. (2008). Diagnosis and treatment of dementia: 5. Nonpharmacologic and pharmacologic therapy for mild to moderate dementia. Canadian Medical Association Journal, 179(10), 1019-1026.

Knapp, M. J., Knopman, D. S., Solomon, P. R., Pendlebury, W. W., Davis, C. S. \& Gracon, S. I. (1994). A 30-week randomized controlled trial of high-dose tacrine in patients with Alzheimer's disease. Journal of the American Medical Association, 271(13), 985-991.

Knapp, M., Thorgrimsen, L., Patel, A., Spector, A., Hallam, A., Woods, B., et al. (2006). Cognitive stimulation therapy for people with dementia: Cost-effectiveness analysis. British Journal of Psychiatry, $188,574-580$.

Lezak, M. (2004). Neuropsychological Assessment (4a. ed.). New York: Oxford University Press.

Loewen, S. \& Anderson, B. (1988). Reliability of the Modified Motor Assessment Scale and the Barthel Index. Physical Therapy, 68(7), 1077-1081.

Logsdon, R. G., Gibbons, L. E., McCurry, S. M. \& Teri, L. (1999). Quality of life in Alzheimer's disease: Patient and caregiver reports. Journal of Mental Health and Aging, 5(1), 21-32.

Mahoney, F. I. \& Barthel, D. W. (1965). Functional evaluation: The Barthel Index. Maryland State Medical Journal, 14, 56-61.

Moniz-Cook, E., Vernooij-Dassen, M., Woods, B. \& Orrell, M. (2011). Psychosocial interventions in dementia care research: The INTERDEM manifesto. Aging Ë Mental Health, 15(3), 283-290.

Moniz-Cook, E., Vernooij-Dassen, M., Woods, R., Verhey, F., Chattat, R., de Vugt, M., et al. (2008). A European consensus on outcome measures for psychosocial intervention research in dementia care. Aging E⿱ Mental Health, 12(1), 14-29.

Neal, M. \& Briggs, M. (2003). Validation therapy for dementia. Cochrane Database of Systematic Reviews, 3, Artículo CD001394. Recuperado el 12 de marzo del 2011, de http://www.ncbi.nlm.nih. gov/pubmed/12917907

Olazarán, J., Reisberg, B., Clare, L., Cruz, I., Peña-Casanova, J., del Ser, T., et al. (2010). Nonpharma- cological therapies in Alzheimer's disease: A systematic review of efficacy. Dementia and Geriatric Cognitive Disorders, 30(2), 161-178.

Olin, J. \& Schneider, L. (2002). Galantamine for Alzheimer's disease. Cochrane Database of Systematic Reviews, 3, CD001747.

Oliver, A., Navarro, E., Meléndez, J., Molina, C. \& Tomás, J. (2009). Modelo de ecuaciones estructurales para predecir el bienestar y la dependencia funcional en adultos mayores de la República Dominicana. Revista Panamericana de Salud Pública, 26(3), 189-196.

Orrell, M., Spector, A., Thorgrimsen, L. \& Woods, B. (2005). A pilot study examining the effectiveness of Maintenance Cognitive Stimulation Therapy (MTEC) for people with dementia. International Journal of Geriatric Psychiatry, 20(5), 446-451.

Peña-Casanova, J. (1999). Tratamiento no farmacológico (cognitivo). En J. Peña-Casanova (Ed.), Enfermedad de Alzheimer. Del diagnóstico a la terapia: conceptos y hechos (pp. 94-98). Barcelona: Fundación La Caixa.

Quiroga, P., Albala, C. \& Klaasen, G. (2004). Validation of a screening test for age associated cognitive impairment, in Chile. Revista Médica de Chile, 132(4), 467-78.

Rabins, P. V., Blacker, D., Rovner, B. W., Rummans, T., Schneider, L. S., Tariot, P. N., et al. (2007). American Psychiatric Association practice guideline for the treatment of patients with Alzheimer's disease and other dementias. American Journal of Psychiatry, 164(12), 5-56.

Rosen, W. G., Mohs, R. C. \& Davis, K. L. (1984). A new rating scale for Alzheimer disease. American Journal of Psychiatry, 141(11), 1356-1364.

Sousa, R. M., Ferri, C., Acosta, D., Albanese, E., Guerra, M., Huang, Y., et al. (2009). Contribution of chronic diseases to disability in elderly people in countries with low and middle incomes: A 10/66 Dementia Research Group population-based survey. Lancet, 374(9704), 1821-1830.

Spector, A., Orrell, M., Davies, S. \& Woods, B. (2001). The development of an evidence based package of psychological therapies for dementia groups. Neuropsychological Rehabilitation, 11(3-4), 377-397.

Spector, A., Orrell, M. \& Woods, B. (2010). Cognitive Stimulation Therapy (TEC): Effects on different 
areas of cognitive function for people with dementia. International Journal of Geriatric Psychiatry, 25(12), 1253-1258.

Spector, A., Thorgrimsen, L., Woods, B. \& Orrell, M. (2006). Making a difference: An evidence-based group programme to offer Cognitive Stimulation Therapy (CST) to people with dementia. Manual for group leaders. United Kingdom: Hawker Publications.

Spector, A., Thorgrimsen, L., Woods, B., Royan, L., Davies, S., Butterworth, M., et al. (2003). Efficacy of an evidence-based cognitive stimulation therapy programme for people with dementia: Randomised controlled trial. British Journal of Psychiatry, 183, 248-254.

Sramek, J. J. \& Cutler, N. R. (1999). Recent developments in the drug treatment of Alzheimer's disease. Drugs and Aging, 14(5), 359-373.

Tárraga, L., Boada, M., Modinos, G., Espinosa, A., Diego, S., Morera, A., et al. (2006). A randomised pilot study to assess the efficacy of an interactive, multimedia tool of cognitive stimulation in Alzheimer's disease. Journal of Neurology, Neurosurgery $\mathcal{E}$ Psychiatry, 77(10), 1116-1121.

Uzzell, B. \& Gross, T. (1986). Clinical neuropsychology of intervention. Boston: Martinus Nijhoff.

Vasse, E., Vernooij-Dassen, M., Cantegril, I., Franco, M., Dorenlot, P., Woods, B., et al. (2012). Guidelines for psychosocial interventions in dementia care: A European survey and comparison. International Journal of Geriatric Psychiatry, 27(1), 40-48.

Verhey, F. R., Houx, P., Van Lang, N., Huppert, F., Stoppe, G., Saerens, J., et al. (2004). Cross-national comparison and validation of the Alzheimer's Disease Assessment Scale: Results from the European Harmonization Project for Instruments in Dementia (EURO-HARPID). International Journal of Geriatric Psychiatry, 19(1), 41-50.

Vernooij-Dassen, M., Vasse, E., Zuidema, S., CohenMansfield, J. \& Moyle, W. (2010). Psychosocial interventions for dementia patients in long-term care. International Psychogeriatrics, 22(7), 1121 1128.

Whitehead, A., Perdomo, C., Pratt, R., Birks, J., Wilcock, G. \& Grimley, J. (2004). Donepezil for the symptomatic treatment of patients with mild to moderate Alzheimer's disease: A meta-analysis of individual patient data from randomised controlled trials. International Journal of Geriatric Psychiatry, 19(7), 624-633.

Woods, B., Aguirre, E., Spector, A. E. \& Orrell, M. (2012). Cognitive stimulation to improve cognitive functioning in people with dementia. Cochrane Database of Systematic Reviews, 2, Artículo CD005562. Recuperado el 20 de marzo del 2012, de http:// onlinelibrary.wiley.com/doi/10.1002/14651858. CD005562.pub2/full

Woods, B., Spector, A., Jones, C., Orrell, M. \& Davies, D. (2005). Reminiscence therapy for dementia. Cochrane Database of Systematic Reviews, 2, Artículo CD001120. Recuperado el 20 de marzo de 2012, de http://onlinelibrary.wiley.com/ doi/10.1002/14651858.CD001120.pub2/full

Woods, B., Thorgrimsen, L., Spector, A., Royan, L. \& Orrell, M. (2006). Improved quality of life and cognitive stimulation therapy in dementia. Aging EO Mental Health, 10(3), 219-226.

World Health Organization \& Alzheimer's Disease International. (2012). Dementia. A public health priority. Geneva: World Health Organization Press.

Zamarrón, M. D., Tárraga, L. \& Fernández-Ballesteros, R. (2008). Plasticidad cognitiva en personas con la enfermedad de Alzheimer que reciben programas de estimulación cognitiva. Psicothema, 20(3), 432-437.

Zanetti, O., Binetti, G., Magni, E., Rozzini, L., Bianchetti, A. \& Trabucchi, M. (1997). Procedural memory stimulation in Alzheimer's disease: Impact of a training programme. Acta Neurologica Scandinavica, 95(3), 152-157.

Zanetti, O., Frisoni, G. B., De Leo, D., Dello Buono, M., Bianchetti, A. \& Trabucchi, M. (1995). Reality orientation therapy in Alzheimer disease: Useful or not? A controlled study. Alzheimer Disease and Associated Disorders, 9(3), 132-138.

Zanetti, O., Zanieri, G., Di Giovanni, G., De Vreese, L., Pezzini, A., Metitieri, T., et al. (2001). Effectiveness of procedural memory stimulation in mild Alzheimer's disease patients: A controlled study. Neuropsychological Rehabilitation, 11(3-4), 263-272. 
